\title{
Methicillin-Resistant Staphylococcus aureus in the Neonatal Intensive Care Unit
}

\author{
Melissa U. Nelson, MD and Patrick G. Gallagher, MD \\ Division of Perinatal Medicine, Department of Pediatrics, Yale University School of Medicine, New \\ Haven, Connecticut, USA
}

\begin{abstract}
Methicillin-resistant Staphylococcus aureus is a frequent source of infections affecting premature and critically ill infants in neonatal intensive care units. Neonates are particularly vulnerable to colonization and infection with Methicillin-resistant Staphylococcus aureus, and many studies have attempted to identify risk factors that predispose certain infants to its acquisition in order to discover potential areas for clinical intervention. In addition, epidemiologic assessment of transmission patterns and molecular analysis of changes in the characteristics of Methicillinresistant Staphylococcus aureus strains over time have helped clarify additional factors affecting Methicillin-resistant Staphylococcus aureus infections in the neonatal intensive care unit. Numerous strategies for prevention and eradication have been employed with variable rates of success. Despite these interventions, Methicillin-resistant Staphylococcus aureus remains a significant source of morbidity in the neonatal intensive care unit population.
\end{abstract}

\section{INTRODUCTION}

Methicillin-resistant Staphylococcus aureus (MRSA) was first isolated in hospitals in the United Kingdom in $1961 .{ }^{1}$ Since the description of that initial case, MRSA has become increasingly prevalent and is now an increasing cause of infections worldwide. The proportion of healthcare-associated staphylococcal infections in United States' intensive care units due to MRSA has continued to rise, from $35.9 \%$ in 1992 to $64.4 \%$ in $2003 .{ }^{2}$

Twenty years after the first MRSA case was described, the first neonatal case of MRSA infection occurring in a neonatal intensive care unit (NICU) was published. ${ }^{3}$ Since that time, MRSA has become a frequent source of infections affecting premature and critically ill neonates in NICUs. ${ }^{4-10}$ Infants seem to be particularly vulnerable to colonization and infection with MRSA, and many studies have attempted to ascertain which risk factors might predispose certain infants to these outcomes. ${ }^{4,11-14}$ MRSA colonization or infection in neonates is associated with significant morbidity, and the cost of medical treatment for affected infants carries a high financial burden. ${ }^{15}$ Due to the serious consequences that are associated with MRSA infections in neonates, much effort has been made to prevent and control epidemics in NICUs.

\footnotetext{
(c) 2012 Elsevier Inc. All rights reserved.

Address correspondence to: Patrick G. Gallagher, Department of Pediatrics, Yale University School of Medicine, 333 Cedar Street, P. O. Box 208064, New Haven, CT 06520-8064, USA. Tel: (203) 688-2896; Fax: (203) 785-6974; patrick.gallagher@yale.edu.

Publisher's Disclaimer: This is a PDF file of an unedited manuscript that has been accepted for publication. As a service to our customers we are providing this early version of the manuscript. The manuscript will undergo copyediting, typesetting, and review of the resulting proof before it is published in its final citable form. Please note that during the production process errors may be discovered which could affect the content, and all legal disclaimers that apply to the journal pertain.
} 


\section{EPIDEMIOLOGY OF MRSA}

\section{NEONATAL ACQUISITION OF MRSA}

Neonates are exposed to Staphylococcus aureus shortly after birth and can become colonized quickly after contact with adult skin or their environment. ${ }^{16,17}$ Between 30-70\% of humans are carriers of Staphylococcus aureus, so neonates have a very high likelihood of exposure during the immediate period after birth. ${ }^{17}$ Most common sites of colonization with $S$. aureus include the umbilical cord, skin, nasopharynx, and gastrointestinal tract. ${ }^{16,17}$ For MRSA, the nares and umbilicus are the most common sites of initial colonization. ${ }^{12}$

Neonates can become colonized by MRSA in a number of different ways. Traditionally, MRSA has been demonstrated to spread horizontally by healthcare-associated transmission, i.e. via contact with healthcare workers or the hospital environment. ${ }^{8,18,19}$ Additional factors, such as overcrowding and understaffing in the NICU, have been associated with increased risk of healthcare-associated transmission and colonization, which may lead to epidemics of MRSA infection. ${ }^{20,21}$

Recently, vertical transmission of MRSA from mothers to their infants has been described. ${ }^{10,21}$ One study showed that the vaginal colonization rate of MRSA in pregnant women was $2.8 \%$, which could allow them to act as reservoirs of MRSA with transmission to their offspring during delivery. ${ }^{22}$ Another report described maternal vertical transmission of MRSA, with maternal MRSA chorioamnionitis associated with neonatal MRSA sepsis. ${ }^{23}$ Vertical transmission was also described in a case of a mother who had MRSA colonization of her nares and subsequently transmitted MRSA to three of her four quadruplets. ${ }^{24}$ Mothers have also been shown to vertically transmit MRSA to their infants via their breast milk. ${ }^{25,26}$ Fathers have spread MRSA by vertical transmission through direct contact with their infants. ${ }^{27}$

Finally, horizontal transmission from siblings and other contacts is another way that neonates can become colonized or infected with MRSA. One study showed that multiple gestation status was independently associated with increased risk of MRSA colonization in neonates. ${ }^{13}$ Another study found that uncolonized patients in a NICU have a higher risk of MRSA colonization when they have a MRSA-colonized sibling also admitted simultaneously in the NICU. ${ }^{19}$

\section{PREVALENCE OF MRSA IN NICUs}

The prevalence of MRSA colonization and infection rates in NICUs varies widely from institution to institution due to many different factors including differences in hospital infection control policies, differences in MRSA prevalence in the surrounding local communities, and differences in worldwide MRSA colonization rates. Additionally, accurate measures of prevalence are further complicated based on whether MRSA prevalence rates are calculated during endemic versus epidemic time periods. Prevalence rates are often measured during a MRSA outbreak in a NICU, and these increased epidemic rates can potentially skew assumptions about true baseline endemic rates. Despite these variations in prevalence measurements, studies have reported anywhere between $0.6 \%-8.4 \%$ of NICU patients being colonized or infected with MRSA during their study periods. $4,7,10,12,14,15,28$

In an attempt to better assess the scope and magnitude of late-onset MRSA infections in United States' NICUs, one study specifically looked at the rate of infections occurring after three days of life attributed to MRSA in NICUs participating in the National Nosocomial Infections Surveillance System over a ten-year study period. ${ }^{6}$ They found that the incidence of MRSA infections increased by $308 \%$ from 0.7 to 3.1 per 10,000 patient-days between 1995 and 2004. ${ }^{6}$ This study's results were taken from a broad sample of 149 NICUs 
throughout the United States and provide further support that rates of MRSA-related infections are steadily increasing in this nation's NICUs.

\section{RISK FACTORS FOR MRSA COLONIZATION AND INFECTION IN NEONATES}

Studies have described a variety of risk factors that predispose infants to colonization and infection with MRSA. Low birth weight has been shown to be associated with increased risk of MRSA colonization and/or infection in multiple studies. ${ }^{4,11-14}$ Prematurity and younger gestational age have also been shown to be risk factors for MRSA colonization and infection, ${ }^{12,13}$ as has multiple gestations. ${ }^{13} \mathrm{~A}$ variety of procedures and devices that neonates in NICUs often require during their hospital stay are also associated with increased risk of MRSA, including endotracheal tube intubation and mechanical ventilation, ${ }^{11,13}$ percutaneous central venous catheterization, ${ }^{11}$ and surgery. ${ }^{4}$ Feeding methods, including gavage feedings ${ }^{13}$ and parenteral feedings, ${ }^{11}$ have also been associated with higher risk of MRSA infection. Longer length of hospital stay, ${ }^{13}$ kangaroo mother care, ${ }^{14}$ and presence of eye mucous in neonates' eyes ${ }^{14}$ have been shown to be independent risk factors for MRSA infection.

Most importantly, several studies have shown that neonatal colonization with MRSA is a risk factor for subsequent development of MRSA infection. ${ }^{12,14}$ Huang et al found that infants who were colonized with MRSA had a significantly higher rate of MRSA infection (26\%) as compared to those who were not colonized ( $2 \%) .{ }^{12}$

\section{EVOLUTION OF MRSA STRAINS}

Over the years, new MRSA strains have emerged and become significant sources of infection for patients in the community and in the hospital. Historically, most MRSA infections were due to healthcare-associated MRSA strains (HA-MRSA), which were spread by transmission in the hospital setting. For the first time in the 1990s, adult and pediatric patients without histories of hospitalization or without risk factors for encountering HAMRSA were found to have MRSA colonization or infection. ${ }^{29,30}$ Further analyses of these strains by molecular methods led to the discovery that distinct strains of MRSA exist in the community as well, and these strains became known as community-associated MRSA (CAMRSA).

HA-MRSA and CA-MRSA have been shown to be genotypically and phenotypically distinct (Table 1). ${ }^{31}$ HA-MRSA and CA-MRSA carry different staphylococcal chromosome cassette (SCC) mecA types that encode for the penicillin-binding protein $2 \mathrm{~A}$ and confer distinct patterns of antibiotic resistance. ${ }^{31}$ While HA-MRSA clones are associated with SCC mec $A$ types I - III, CA-MRSA clones are associated with SCC mec $A$ types IV-V. ${ }^{31,32}$ Healthcare-associated strains tend to be resistant to multiple antibiotics, whereas community-associated strains are often susceptible to several non- $\beta$-lactam antibiotics such as trimethoprim-sulfamethoxazole, clindamycin, and quinolones. ${ }^{32,33}$ Additionally, CAMRSA strains are more often associated with expression of Panton-Valentine leukocidin (PVL), which leads to production of cytotoxins that cause cell lysis and tissue necrosis. ${ }^{31,32,34}$ Community-associated strains are also more often associated with skin and soft tissue infections as compared to healthcare-associated strains. ${ }^{31}$

Infections with HA-MRSA and newly emerging strains of CA-MRSA have also been observed in neonates in the NICU. Healy et al published what they reported to be the first documentation of CA-MRSA infections in neonates who were hospitalized since birth in a NICU in 2004. ${ }^{5}$ Gregory et al published similar findings that noted a shift from predominately HA-MRSA infections in their NICU between the years 2000-2004 and then CA-MRSA between 2006-2007. ${ }^{28}$ Carey et al witnessed a similar change in the types of 
MRSA infections in their NICU, as molecular analysis demonstrated a change from mainly HA-MRSA infection and colonization between the years 2001-2004 and then CA-MRSA between 2005-2008. ${ }^{35}$ Several other reports have described similar MRSA infection outbreaks specifically associated with one common type of CA-MRSA, pulse-field type USA300, in the newborn nursery and NICU. 7,36 A prospective study by Seybold et al sought to look more closely at the impact of CA-MRSA colonization and infection in their NICU and to uncover its potential modes of transmission. ${ }^{10}$ They found that rates of CAMRSA were increasing over time within their unit and that CA-MRSA was acquired during birth whereas HA-MRSA was transmitted nosocomially. 10

\section{BURDEN OF MRSA DISEASE MRSA ASSOCIATED MORBIDITY AND MORTALITY}

MRSA infection is associated with significant morbidity in the neonatal population. In general, MRSA can cause a variety of serious infections in the pediatric population including blood stream infections, skin and soft tissue infections, conjunctivitis, osteomyelitis, septic arthritis, endocarditis, surgical site infections, meningitis, brain abscesses, pneumonia, and respiratory tract infections. ${ }^{6,33,37}$ These infections can lead to significant morbidity including prolonged hospitalizations, increased need for aggressive treatments, and potential long-term medical problems for affected patients. Based on the serious concern for the rising incidence and mortality of MRSA colonization or infection in children, Song et al performed a study to assess the clinical impact of MRSA colonization or infection on infants in their NICU. ${ }^{15}$ They used a time-dependent proportional hazard model to specifically examine the association between MRSA colonization or infection and mortality, and found that although MRSA colonization or infection was associated with significant morbidity, there was not a significantly associated increased risk of mortality in their NICU. ${ }^{15}$ The rate of mortality for patients with MRSA was $17.8 \%$ as compared to $11.5 \%$ for patients without MRSA colonization or infection, which was not a statistically significant difference. 15

\section{MRSA ASSOCIATED ECONOMIC IMPACT}

Neonatal colonization or infection with MRSA is also associated with an expensive financial burden. Increased rates of MRSA infection can cause increased medical costs due to the price of additional medical treatment and healthcare expenses for methods of screening for and eradicating colonization. Several studies have looked at the economic impact of MRSA colonization and infection in NICUs. Schultz et al sought to examine the effect that MRSA colonization had on total hospital cost. ${ }^{38}$ They retrospectively compared total lengths of stay and hospital costs between MRSA colonized $(n=59)$ and non-colonized $(n=1701)$ neonates in their NICU, and found that although there was no difference between each group of patients' mean daily hospital costs, the MRSA colonized neonates had much longer lengths of hospital admission and a total excess cost of \$6,901,180. ${ }^{38}$ Another study by Song et al looked at the economic impact of neonatal MRSA colonization or infection by performing a retrospective case-control study to compare length of stay and hospital charges between neonates who were colonized or infected with MRSA and those who were not. 15 They found that neonatal MRSA infection was associated with a 40-day increase in length of hospital stay and an increased cost of $\$ 164,301$ per patient. 15

\section{TREATMENT OF MRSA INFECTIONS}

By definition, methicillin-resistant Staphylococcus aureus (MRSA) is a bacterial pathogen that is resistant to methicillin and all $\beta$-lactam antibiotics. Whereas HA-MRSA strains are more often resistant to multiple types of antibiotics, CA-MRSA strains are often susceptible to several non- $\beta$-lactam antibiotics including trimethoprim-sulfamethoxazole, clindamycin, 
and quinolones. ${ }^{32,33}$ According to the American Academy of Pediatrics' Committee on Infectious Diseases' most recent recommendations, empiric antibiotic therapy of lifethreatening infections due to Staphylococcus aureus infection of unknown susceptibility include parenterally administered vancomycin plus nafcillin or oxacillin plus gentamicin (Table 2). ${ }^{39}$ If the patient has recently received several courses of vancomycin and could therefore harbor vancomycin-resistant MRSA, then linezolid could be substituted for vancomycin. Antibiotic recommendations for nonlife-threatening Staphylococcus aureus infections in communities with high rates of MRSA colonization and infection include clindamycin or vancomycin depending on the local rates of clindamycin resistance. Infections due to multi-drug resistant HA-MRSA strains should be treated with a combination of parenteral vancomycin plus gentamicin and/or rifampin, but alternatives such as trimethoprim-sulfamethoxazole, linezolid, quinupristin-dalfopristin, or fluoroquinolones could also be considered pending results of susceptibility testing. Finally, life-threatening infections due to CA-MRSA strains should be treated with a combination of parenteral vancomycin plus gentamicin and/or rifampin, whereas nonlife-threatening infections due to CA-MRSA strains should be treated with clindamycin, trimethoprimsulfamethoxazole, or vancomycin. ${ }^{39}$

In addition to antibiotic treatment, other approaches such as the use of anti-staphylococcal immunoglobulins have been attempted to try to prevent Staphylococcus aureus infections. ${ }^{40}$ A Cochrane review by Shah et al reviewed the literature regarding the safety and efficacy of administration of anti-staphylococcal immunoglobulins for the prevention of staphylococcal infections in very low birth weight (VLBW) neonates. Although the initial results in animal studies appeared promising, this meta-analysis of three studies involving treatment of greater than 2,700 neonates showed no significant difference in the prevention of staphylococcal infections in VLBW infants after administration of anti-staphylococcal immunoglobulins. Therefore, they concluded that this treatment option should not be recommended currently for prevention of staphylococcal infections in preterm or VLBW neonates and that further research should be conducted to investigate the efficacy of other types of anti-staphylococcal immunoglobulins. ${ }^{40}$

\section{METHODS OF MRSA ERADICATION AND ELIMINATION}

Various effective methods of infection control have been utilized to help reduce the incidence of MRSA infections. In the hospital setting, studies have looked at the effectiveness of various practices, including surveillance screening, nursing workload, and hand hygiene, and found that increased rates of hand washing and screening patients for MRSA at admission are the most effective strategies to reduce rates of MRSA transmission among hospitalized patients. ${ }^{41}$

Many different interventions have been utilized to halt outbreaks of MRSA within the NICU. A NICU in Japan reported successful eradication of an outbreak of MRSA through implementation of standard practice measures including improved hand hygiene and use of disposable rubber gloves and gowns for patient care as well as patient decolonization with intranasal application of mupirocin ointment, bathing in diluted povidone iodine solution, and intraoral application of methylrosaniline chloride. ${ }^{21}$ Another NICU in New York reported adopting methods that included surveillance cultures of patients, staff, and environment, strict contact precautions, cohorting of patients and nurses, molecular typing of strains, and decolonization of patients with mupirocin and staff members with mupirocin and hexachlorophene showers in an effort to stop a MRSA outbreak within their unit. ${ }^{8}$ Still another NICU in St. Louis reported successful elimination of a MRSA outbreak within their unit via active surveillance of patients and staff, improved hygiene practices, contact isolation, cohorting, decolonization of affected patients with mupirocin ointment, and 
decolonization of affected staff with hexachlorophene showers as well as administration of rifampin, trimethoprim-sulfamethoxazole, and mupirocin. ${ }^{13}$ Another NICU in Switzerland utilized active surveillance of patients, contact isolation, and molecular analysis of positive case strains to help identify the sources of MRSA in their unit and stop further outbreak. ${ }^{9}$ In an effort to monitor and reduce MRSA colonization rates within their unit, another NICU in Boston implemented a universal screening program with weekly surveillance culture monitoring and isolation of affected neonates and found that these efforts had variable effects on MRSA incidence. ${ }^{28}$ Finally, a NICU in France reported attempting various practices, such as systematic screening of patients, barrier precautions, cohorting, isolation, hand hygiene observation, staff training, and decolonization of patients and staff, to stop an outbreak within their unit, but only found that the hand hygiene monitoring and cohorting of patients and staff seemed to help control the outbreak. ${ }^{42}$

Although numerous methods with variable success rates have been published regarding attempts to eradicate MRSA outbreaks in NICUs, a consensus for guidelines was lacking. In response to this lack of universal recommendations, Gerber et al published a consensus statement in 2006 with guidelines for the prevention and control of MRSA colonization and infection (Table 3). ${ }^{11}$ They convened a group of infectious disease physicians and infection control experts from several NICUs in the Chicago area to look at data from MRSA outbreaks and assess the effectiveness of various control methods in order to create a set of standard guidelines. Their thorough recommendations included improved hand hygiene practices, cohorting and isolation of patient and staff, neonatal surveillance cultures, screening of healthcare workers during outbreaks, decolonization practices, environmental cultures, molecular analysis of strains during outbreaks, open-communication between NICUs, collaboration with hospitals and public health institutions, and upholding regulations and standards for staffing and unit censuses. ${ }^{11}$ They acknowledged that while local infection control practices at individual institutions might help reduce infection rates at their own institution, they would not have a significant effect on reducing the worldwide increased prevalence of MRSA. Therefore, they developed their standard guidelines for the prevention and control of MRSA outbreaks in hopes that a broader effort might exert more widespread MRSA control.

\section{MRSA AND NICUs: LOOKING FORWARD}

MRSA continues to be a significant source of morbidity for the NICU population. The historical trends of increasing prevalence of MRSA in NICUs and evolution of more MRSA strain types within the hospital and community settings suggest that clinical struggles with this pathogen will continue in the future. As the population of NICU patients increases, due to limits of viability being pushed to even younger gestational ages and rapidly advancing technologies enabling more babies to survive, there is potential that many more neonates will be at risk for colonization and infection with MRSA. Furthermore, emergence of multidrug resistant MRSA strains that are even resistant to the longstanding treatment mainstay, vancomycin, suggest that difficulties treating MRSA infections will only continue to escalate.

As MRSA infections continue to cause increasing morbidity in the NICU, it is imperative that future research both evaluates the effectiveness of current interventions and also develops new strategies for eliminating MRSA colonization and infections in neonates. Clinical trials should be conducted to evaluate the efficacy of MRSA eradication strategies previously reported in case reports, such as cohorting and isolation, surveillance cultures, and decolonization. Research in academic centers, biotechnology, and pharmaceutical companies should focus on implementing a multidisciplinary approach for the development of new antibiotics against MRSA as strains resistant to current antibiotic regimens continue 
to emerge. ${ }^{43}$ Additional treatment strategies, including innate immune-enhancement strategies ${ }^{44}$ and genetic modulation of MRSA to restore antibiotic susceptibility, ${ }^{45}$ that have already been explored in vitro and in vivo in animal models should be further evaluated as potential supplemental interventions for halting MRSA infections in human subjects. Finally, it is critical that medical communities continue to monitor and share reports regarding their local prevalence of MRSA strains, incidence of invasive MRSA infections, and MRSA antibiotic susceptibility patterns in an effort to provide cumulative data that can ultimately contribute to global awareness and treatment guidelines for this pervasive pathogen.

\section{Acknowledgments}

Supported in part by T32 HD07094 (MUN).

\section{References}

1. Jevons M. “Cellbenin”-resistant staphylococci. BMJ. 1961; 1:124-125.

2. Klevens RM, Edwards JR, Tenover FC, et al. Changes in the epidemiology of methicillin-resistant Staphylococcus aureus in intensive care units in US hospitals, 1992-2003. Clin Infect Dis. 2006; 42:389-391. [PubMed: 16392087]

3. Weeks JL, Garcia-Prats JA, Baker CJ. Methicillin-resistant Staphylococcus aureus osteomyelitis in a neonate. JAMA. 1981; 245:1662-1664. [PubMed: 6907329]

4. Carey AJ, Duchon J, Della-Latta P, et al. The epidemiology of methicillin-susceptible and methicillin-resistant Staphylococcus aureus in a neonatal intensive care unit, 2000-2007. J Perinatol. 2010; 30:135-139. [PubMed: 19710681]

5. Healy CM, Hulten KG, Palazzi DL, et al. Emergence of new strains of methicillin-resistant Staphylococcus aureus in a neonatal intensive care unit. Clin Infect Dis. 2004; 39:1460-1466. [PubMed: 15546082]

6. Lessa FC, Edwards JR, Fridkin SK, et al. Trends in incidence of late-onset methicillin-resistant Staphylococcus aureus infection in neonatal intensive care units: data from the National Nosocomial Infections Surveillance System, 1995-2004. Pediatr Infect Dis J. 2009; 28:577-581. [PubMed: 19478687]

7. McAdams RM, Ellis MW, Trevino S, et al. Spread of methicillin-resistant Staphylococcus aureus USA300 in a neonatal intensive care unit. Pediatrics International. 2008; 50:810-815. [PubMed: 19067897]

8. Saiman L, Cronquist A, Wu F, et al. An outbreak of methicillin-resistant Staphylococcus aureus in a neonatal intensive care unit. Infect Control Hosp Epidemiol. 2003; 24:317-321. [PubMed: 12785403]

9. Sax H, Posfay-Barbe K, Harbarth S, et al. Control of a cluster of community-associated, methicillinresistant Staphylococcus aureus in neonatology. J Hosp Infect. 2006; 63:93-100. [PubMed: 16542756]

10. Seybold U, Halvosa JS, White N, et al. Emergence of and risk factors for methicillin-resistant Staphylococcus aureus of community origin in intensive care nurseries. Pediatrics. 2008; 122:1039-1046. [PubMed: 18977985]

11. Gerber SI, Jones RC, Scott MV, et al. Management of outbreaks of methicillin-resistant Staphylococcus aureus infection in the neonatal intensive care unit: a consensus statement. Infect Control Hosp Epidemiol. 2006; 27:139-145. [PubMed: 16465630]

12. Huang YC, Chou YH, Su LH, et al. Methicillin-resistant Staphylococcus aureus colonization and its association with infection among infants hospitalized in neonatal intensive care units. Pediatrics. 2006; 118:469-474. [PubMed: 16882797]

13. Khoury J, Jones M, Grim A, et al. Eradication of methicillin-resistant Staphylococcus aureus from a neonatal intensive care unit by active surveillance and aggressive infection control measures. Infect Control Hosp Epidemiol. 2005; 26:616-621. [PubMed: 16092741] 
14. Sakaki H, Nishioka M, Kanda K, et al. An investigation of the risk factors for infection with methicillin-resistant Staphylococcus aureus among patients in a neonatal intensive care unit. Am J Infect Control. 2009; 37:580-586. [PubMed: 19535174]

15. Song X, Perencevich E, Campos J, et al. Clinical and economic impact of methicillin-resistant Staphylococcus aureus colonization or infection on neonates in intensive care units. Infect Control Hosp Epidemiol. 2010; 31:177-182. [PubMed: 20001732]

16. Bizzarro MJ, Gallagher PG. Antibiotic-resistant organisms in the neonatal intensive care unit. Semin Perinatol. 2007; 31:26-32. [PubMed: 17317424]

17. Cimolai N. Staphylococcus aureus outbreaks among newborns: new frontiers in an old dilemma. American Journal of Perinatology. 2003; 20:125-136. [PubMed: 12802712]

18. Eveillard M, Martin Y, Hidri N, et al. Carriage of methicillin-resistant Staphylococcus aureus among hospital employees: prevalence, duration, and transmission to households. Infect Control Hosp Epidemiol. 2004; 25:114-120. [PubMed: 14994935]

19. Geva A, Wright SB, Baldini LM, et al. Spread of methicillin-resistant Staphylococcus aureus in a large tertiary NICU: network analysis. Pediatrics. 2011; 128:e1173-1180. [PubMed: 22007011]

20. Andersen BM, Lindemann R, Bergh K, et al. Spread of methicillin-resistant Staphylococcus aureus in a neonatal intensive unit associated with understaffing, overcrowding and mixing of patients. J Hosp Infect. 2002; 50:18-24. [PubMed: 11825047]

21. Shiojima T, Ohki Y, Nako Y, et al. Immediate control of a methicillin-resistant Staphylococcus aureus outbreak in a neonatal intensive care unit. Journal of Infection and Chemotherapy. 2003; 9:243-247. [PubMed: 14513393]

22. Chen KT, Huard RC, Della-Latta P, et al. Prevalence of methicillin-sensitive and methicillinresistant Staphylococcus aureus in pregnant women. Obstetrics and Gynecology. 2006; 108:482487. [PubMed: 16946204]

23. Pimentel JD, Meier FA, Samuel LP. Chorioamnionitis and neonatal sepsis from communityassociated MRSA. Emerg Infect Dis. 2009; 15:2069-2071. [PubMed: 19961709]

24. Morel AS, Wu F, Della-Latta P, et al. Nosocomial transmission of methicillin-resistant Staphylococcus aureus from a mother to her preterm quadruplet infants. Am J Infect Control. 2002; 30:170-173. [PubMed: 11988712]

25. Behari P, Englund J, Alcasid G, et al. Transmission of methicillin-resistant Staphylococcus aureus to preterm infants through breast milk. Infect Control Hosp Epidemiol. 2004; 25:778-780. [PubMed: 15484804]

26. Gastelum DT, Dassey D, Mascola L, et al. Transmission of community-associated methicillinresistant Staphylococcus aureus from breast milk in the neonatal intensive care unit. Pediatr Infect Dis J. 2005; 24:1122-1124. [PubMed: 16371885]

27. Al-Tawfiq JA. Father-to-infant transmission of community-acquired methicillin-resistant Staphylococcus aureus in a neonatal intensive care unit. Infect Control Hosp Epidemiol. 2006; 27:636-637. [PubMed: 16755488]

28. Gregory ML, Eichenwald EC, Puopolo KM. Seven-year experience with a surveillance program to reduce methicillin-resistant Staphylococcus aureus colonization in a neonatal intensive care unit. Pediatrics. 2009; 123:e790-796. [PubMed: 19403471]

29. Centers for Disease Control and Prevention. Four pediatric deaths from community-acquired methicillin-resistant Staphylococcus aureus--Minnesota and North Dakota, 1997-1999. JAMA. 1999; 282:1123-1125. [PubMed: 10501104]

30. Herold BC, Immergluck LC, Maranan MC, et al. Community-acquired methicillin-resistant Staphylococcus aureus in children with no identified predisposing risk. JAMA. 1998; 279:593598. [PubMed: 9486753]

31. Diederen BM, Kluytmans JA. The emergence of infections with community-associated methicillin resistant Staphylococcus aureus. The Journal of Infection. 2006; 52:157-168. [PubMed: 16289303]

32. Carey AJ, Long SS. Staphylococcus aureus: a continuously evolving and formidable pathogen in the neonatal intensive care unit. Clin Perinatol. 2010; 37:535-546. [PubMed: 20813269]

33. Carey AJ, Saiman L, Polin RA. Hospital-acquired infections in the NICU: epidemiology for the new millennium. Clin Perinatol. 2008; 35:223-249. [PubMed: 18280884] 
34. Naimi TS, LeDell KH, Como-Sabetti K, et al. Comparison of community- and health careassociated methicillin-resistant Staphylococcus aureus infection. JAMA. 2003; 290:2976-2984. [PubMed: 14665659]

35. Carey AJ, Della-Latta P, Huard R, et al. Changes in the molecular epidemiological characteristics of methicillin-resistant Staphylococcus aureus in a neonatal intensive care unit. Infect Control Hosp Epidemiol. 2010; 31:613-619. [PubMed: 20420500]

36. James L, Gorwitz RJ, Jones RC, et al. Methicillin-resistant Staphylococcus aureus infections among healthy full-term newborns. Arch Dis Child Fetal Neonatal Ed. 2008; 93:F40-44. [PubMed: 17412749]

37. Woodlief RS, Markowitz JE. Unrecognized invasive infection in a neonate colonized with methicillin-resistant Staphylococcus aureus. J Pediatr. 2009; 155:943-943. e941. [PubMed: 19914437]

38. Schultz ED, Tanaka DT, Goldberg RN, et al. Effect of methicillin-resistant Staphylococcus aureus colonization in the neonatal intensive care unit on total hospital cost. Infect Control Hosp Epidemiol. 2009; 30:383-385. [PubMed: 19222371]

39. Pickering, LK.; Baker, CJ.; Kimberlin, DW., et al., editors. Red Book: Report of the Committee on Infectious Diseases. 28. Elk Grove Village, IL: American Academy of Pediatrics; 2009. American Academy of Pediatrics Committee on Infectious Diseases: Staphylococcal Infections; p. 601-615.

40. Shah PS, Kaufman DA. Antistaphylococcal immunoglobulins to prevent staphylococcal infection in very low birth weight infants. Cochrane Database Syst Rev. 2009:CD006449. [PubMed: 19370635]

41. Raboud J, Saskin R, Simor A, et al. Modeling transmission of methicillin-resistant Staphylococcus aureus among patients admitted to a hospital. Infect Control Hosp Epidemiol. 2005; 26:607-615. [PubMed: 16092740]

42. Lepelletier D, Corvec S, Caillon J, et al. Eradication of methicillin-resistant Staphylococcus aureus in a neonatal intensive care unit: which measures for which success? Am J Infect Control. 2009; 37:195-200. [PubMed: 19181423]

43. Morell EA, Balkin DM. Methicillin-resistant Staphylococcus aureus: a pervasive pathogen highlights the need for new antimicrobial development. The Yale Journal of Biology and Medicine. 2010; 83:223-233. [PubMed: 21165342]

44. Rajam G, Hammons GM, Carlone GM, et al. A Novel Innate Immune-Enhancement Strategy Combined with IVIG Rescues Mice from Fatal Staphylococcus aureus Septicemia. International Journal of Microbiology. 2011; 2011:1-5.

45. Tan CM, Therien AG, Lu J, et al. Restoring Methicillin-Resistant Staphylococcus aureus Susceptibility to beta-Lactam Antibiotics. Science Translational Medicine. 2012; 4:126ra135. 
Table 1

Comparison of Healthcare-Associated MRSA (HA-MRSA) and Community-Associated MRSA (CA-MRSA)

\begin{tabular}{lll}
\hline & HA-MRSA & CA-MRSA \\
\hline Clinical spectrum & $\begin{array}{l}\text { Respiratory tract infections } \\
\text { Urinary tract infections } \\
\text { Bloodstream infections }\end{array}$ & Skin and soft tissue infections \\
\hline Resistance pattern & Resistant to multiple antibiotics & Susceptible to multiple antibiotics \\
\hline Genotype & Mainly SCCmec type types I - III & Mainly SCCmec type IV \\
Panton-Valentine leukocidin (PVL) & PVL absent & PVL present \\
\hline
\end{tabular}

Adapted with permission from Elsevier from Diederen et al.(31). 
Table 2

Antimicrobial Agent(s) for the Treatment of Suspected or Proven Methicillin-resistant Staphylococcus aureus (MRSA) Infections

\begin{tabular}{|c|c|c|}
\hline \multirow[t]{4}{*}{ Drugs of choice: } & Vancomycin + nafcillin or oxacillin + gentamicin & $\begin{array}{l}\text { For life-threatening infections; Linezolid could be } \\
\text { substituted for vancomycin if several recent courses } \\
\text { with vancomycin }\end{array}$ \\
\hline & Nafcillin or oxacillin & $\begin{array}{l}\text { For nonlife-threatening infections when community } \\
\text { MRSA rates are low }\end{array}$ \\
\hline & Clindamycin & $\begin{array}{l}\text { For nonlife-threatening infections when MRSA rates } \\
\text { are high and clindamycin resistance is low }\end{array}$ \\
\hline & Vancomycin & $\begin{array}{l}\text { For nonlife-threatening infections when MRSA rates } \\
\text { are high and clindamycin resistance is high }\end{array}$ \\
\hline \multicolumn{3}{|c|}{ II. MRSA Therapy (oxacillin MIC, 4 micrograms/milliliter or greater) } \\
\hline \multicolumn{3}{|c|}{ A. Healthcare-Associated MRSA (Multi-drug Resistant) } \\
\hline Drugs of choice: & Vancomycin \pm gentamicin \pm rifampin & \\
\hline $\begin{array}{l}\text { Alternatives: (susceptibility } \\
\text { results before alternative drugs } \\
\text { used) }\end{array}$ & $\begin{array}{l}\text { Trimethoprim-sulfamethoxazole } \\
\text { Linezolid } \\
\text { Quinupristin-dalfopristin } \\
\text { Fluoroquinolones }\end{array}$ & Not recommended $<18$ years \\
\hline \multicolumn{3}{|c|}{ B. Community-Associated MRSA (Not Multi-drug Resistant) } \\
\hline Drugs of choice: & $\begin{array}{l}\text { Vancomycin } \pm \text { gentamicin (or } \pm \text { rifampin) } \\
\text { Clindamycin (if susceptible) } \\
\text { Trimethoprim-sulfamethoxazole }\end{array}$ & $\begin{array}{l}\text { For life-threatening infections } \\
\text { For pneumonia, septic arthritis, osteomyelitis, or skin } \\
\text { infections } \\
\text { For skin or soft tissue infection }\end{array}$ \\
\hline Alternatives: & Vancomycin & \\
\hline
\end{tabular}


Table 3

Recommendations for Control of Methicillin-resistant Staphylococcus aureus in the Neonatal Intensive Care Unit

\begin{tabular}{|c|c|}
\hline Recommendation Type & Consensus Recommendation \\
\hline Hand Hygiene & $\begin{array}{l}\text { A waterless, alcohol-based hand hygiene product should be available and easily accessible; soap and water should } \\
\text { be used if hands are visibly soiled. } \\
\text { Direct observation of hand hygiene practices and consistent enforcement of proper hand hygiene contribute to } \\
\text { increased rates of compliance. }\end{array}$ \\
\hline Cohorting and Isolation & $\begin{array}{l}\text { MRSA-positive infants should be placed under contact precautions and cohorted; as should the supplies used in } \\
\text { their care. } \\
\text { Gloves and gowns should be worn when caring for MRSA-positive patients. } \\
\text { Masks should be worn for aerosol-generating procedures, such as suctioning. } \\
\text { Whenever possible, nurses should be cohorted for care of MRSA-positive patients. }\end{array}$ \\
\hline Surveillance Cultures & $\begin{array}{l}\text { Infants in the NICU should be screened periodically for MRSA colonization. Screening frequency should be } \\
\text { adjusted based on unit transmission rates (i.e. weekly-monthly). } \\
\text { Culture of nasopharyngeal specimens alone is sufficient. }\end{array}$ \\
\hline Decolonization & Mupirocin may be used for decolonization of infants or healthcare workers if deemed necessary by the institution. \\
\hline Molecular Analysis & $\begin{array}{l}\text { When investigating an outbreak, a molecular epidemiologic tool such as pulse-field gel electrophoresis should be } \\
\text { performed to assess the relatedness of strains found in NICU patients, healthcare workers, and the environment. }\end{array}$ \\
\hline Communication & $\begin{array}{l}\text { Open communication between regional NICUs is essential to prevent spread of MRSA between NICUs when } \\
\text { patients are transferred to different institutions. }\end{array}$ \\
\hline
\end{tabular}

